\title{
Short-term Memory and Sentence Comprehension: A Reply to Vallar and Baddeley, 1987
}

\author{
David Howard and Brian Butterworth \\ University College, London, U.K.
}

What role does a phonological short-term memory store (P.S.T.S.) play in the comprehension of spoken and written sentences? One way to approach this question is to characterise the sentence comprehension performance of subjects who have a P.S.T.S. whose capacity has been reduced or even eliminated by some pathological process. When patients with reduced P.S.T.S. show comprehension deficits, it is tempting to see these as a consequence of the memory problem; and a glance at the literature on such subjects shows that a variety of authors have argued that their patients' comprehension problems are secondary to the memory deficit. The problem with these accounts is simple: when the data are examined in any detail the characteristics of the sentence comprehension deficits show great variation across patients (see, e.g. Butterworth, Campbell, \& Howard, 1986). The obvious possibility is that some or all of these patients have other non-mnestic problems in sentence comprehension, which happen to cooccur with their S.T.M. deficit. We would suggest that a sentence comprehension problem can only be attributed to a P.S.T.S. deficit where:

1. There is evidence that syntactic, lexical, semantic, and pragmatic processes which also contribute to sentence comprehension are not impaired.

2. The characteristics of the comprehension deficit are of a type which would be predicted by an independently motivated theory of the role that phonological processes play.

As Patterson and Coltheart (1987, p.442) put it: "Anyone daring to state that phonology is implicated in comprehension must be prepared to offer very detailed proposals about what sort of phonology or speech related code is meant".

Requests for reprints should be addressed to David Howard, Psychology Department, University College London, Gower Street, London WC1E 6BT, U.K.

(C) 1989 Lawrence Erlbaum Associates Limited 
PV is an Italian patient who, following a left hemisphere C.V.A. in February 1977, had a deficit in short-term memory. She has been very intensively studied; there are three papers which are primarily concerned with her list recall (Basso, Spinnler, Vallar, \& Zanobio, 1982; Vallar \& Baddeley, 1984a; Vallar \& Papagno, 1986), and three which consider her difficulties in sentence comprehension (Vallar \& Baddeley, 1984b; Baddeley, Vallar, \& Wilson, 1987; Vallar \& Baddeley, 1987). In these latter three papers the authors develop the claim that PV's sentence comprehension deficit is best accounted for by her restricted P.S.T.S. They therefore claim that a functioning P.S.T.S. is necessary for normal comprehension of sentences of various kinds-specifically those that PV has difficulty with. These are, Vallar and Baddeley (1987, p.435) suggest, sentences: "(1) when interpretation of lexical semantic and syntactic processing is crucial for comprehension; and (2) when a relatively complete analysis of multiple aspects of the material is needed, and the strategy of concentrating on specific aspects cannot easily be used".

We had earlier reported a woman, RE, who has a developmental restriction of short-term memory (Campbell \& Butterworth, 1985; Butterworth et al., 1986). In list recall tasks, RE could not repeat a list of three digits reliably. Her performance was unaffected by phonological similiarity with spoken and written presentation. Interpreted in terms of Baddeley's (1986) "working memory" model, RE shows no evidence of having any P.S.T.S. at all. If the P.S.T.S. plays a necessary role in (spoken or written) sentence comprehension, RE should have severely impaired understanding of sentences. Yet in a variety of tests of comprehension of written and spoken sentences, RE performed in a way which was indistinguishable from normal controls. This provides very clear counter-evidence to Vallar and Baddeley's assertion that the P.S.T.S. is necessary for sentence comprehension.

Baddeley et al. (1987) and Vallar and Baddeley (1987) are aware of this. Across the two papers they suggest several reasons why RE's performance is irrelevant to any consideration of the normal role of phonological processes in sentence comprehension. Their fundamental objection is that because RE's P.S.T.S. impairment is developmental, she may have developed unusual cognitive abilities to compensate for her memory deficit. They cite three pieces of evidence in support of this:

1. RE has (Vallar \& Baddeley, 1987, p.436): "an auditory memory span of three to four items, despite a total lack of phonological coding in immediate memory. Patients such as PV . . . have a lower immediate memory performance". It is difficult to know quite how to measure span. For instance, according to Basso et al., 1982, PV can only reliably repeat a single digit, but on at least one occasion she repeated a list of five digits. However, a careful reading of the reports on the two subjects reveals that PV's recall 
is better than RE's at least in some tasks. In Fig. 1 we compare PV's and RE's recall of auditorily presented lists of four and five digits (PV data taken from Basso et al., 1982, Fig. 1; RE data from Campbell \& Butterworth, 1985, Fig. 1). It is clear that, in digit recall, PV's performance is if anything rather better than RE's. In both subjects, span varies across tasks and materials. Thus, for example, $\mathrm{PV}$ is able to report only a single digit, letter, or word with perfect reliability in Vallar and Baddeley, (1984a) Table 1, whereas Table 6 shows that she can repeat $100 \%$ of lists of three twosyllable words and of three five-syllable words. RE is more consistent, but shows some variation. Nevertheless, three items appears to be the limit of her span for reliable repetition of letters, digits, or short words. Both PV and RE are reliably better with visual presentation. When, as in the Peterson technique, lists are recalled after a delay filled by irrelevant articulation, normal subjects can recall 2.67 items reliably after a 20 -second delay (Butterworth, Shallice, \& Watson, in press). Under Baddeley's (1986) W.M. model, suppression should have prevented rehearsal of the contents of the P.S.T.S., which should have completely decayed away over this period (Baddeley \& Lewis, 1982). Within this formulation it is difficult to see why a patient whose only W.M. deficit is in the P.S.T.S. can have a span of less than two to three items. We suspect that those patients whose span is reported to be much lower have other processing limitations in speech production, speech comprehension, or repetition which contribute to their reduced repetition spans.

2. Vallar and Baddeley (1987, pp.436-437) state that: "RE's sentence repetition performance, although defective, appears to be superior to that of acquired cases. A measure of RE's sentence span is unfortunately not provided but she is able to repeat 11 out of 40 sentences 15 to 21 words in length". Elaborating on this, Baddeley et al. (1987, p.525) claim that: "the measure of performance most relevant to comprehension in such cases is probably sentence span". We have difficulty with the simple notion of "sentence span". In both normal subjects and patients with short-term memory problems, repetition span for sentences depends not only on their length, but also on their semantic and syntactic structures (eg Saffran \& Marin, 1975; Saffran, Note 1). Any measure of span will therefore depend on the materials used to test for "sentence span".

More importantly, there is a circularity in using "sentence span" to predict sentence comprehension. In coherent texts normal people can, on occasion, repeat 25 words without error (e.g. Wingfield \& Butterworth, 1984), when their span for lists of unrelated words is only 7 or 8 . This difference most plausibly reflects the use of the syntactic and semantic information in the material; this information is clearly the result of applying comprehension processes to the material to be recalled. Thus we would predict that a 


\section{5 digits}
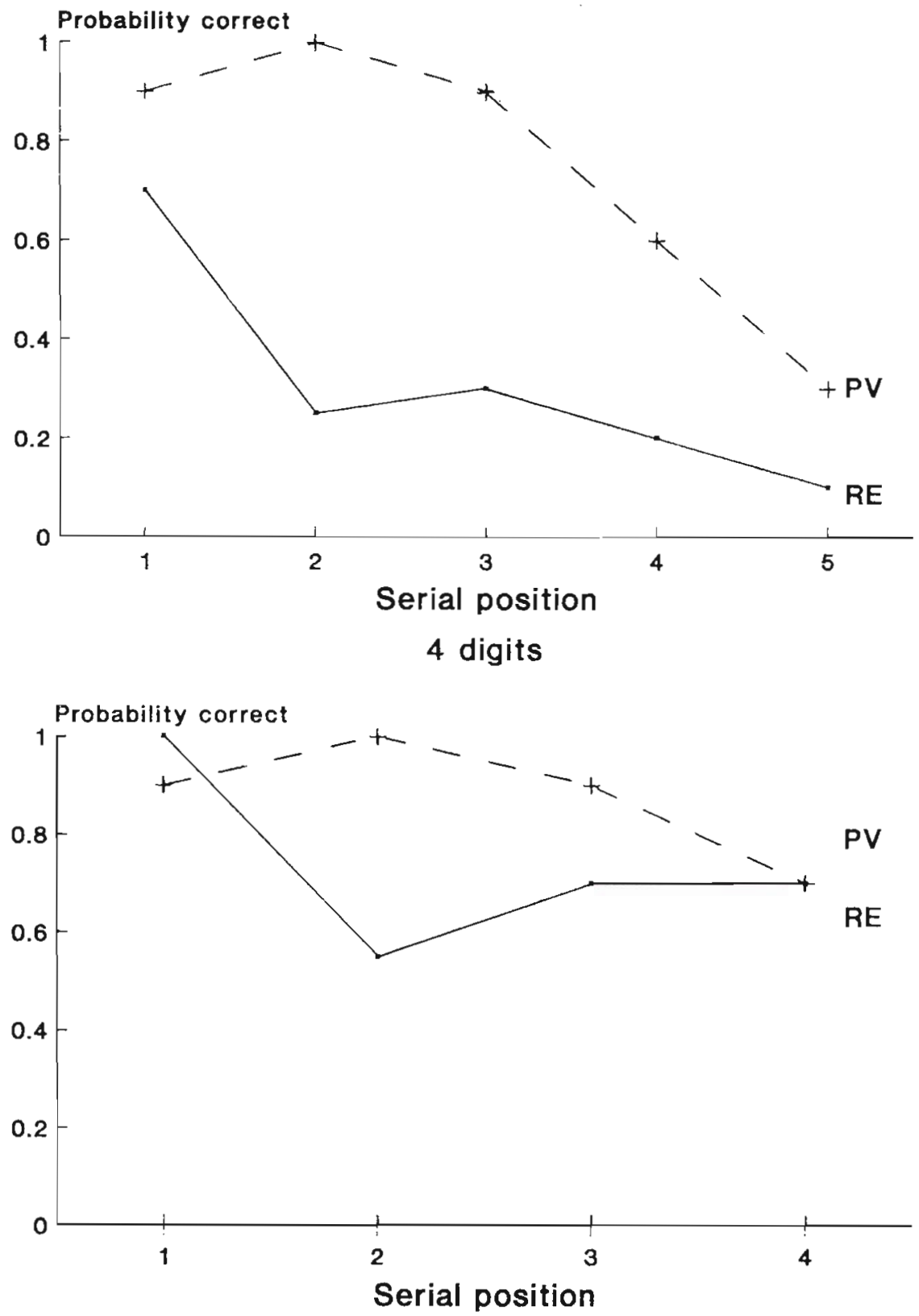

FIG.1 Spoken recall of auditorily presented digits. Data for RE from Campbell and Butterworth, 1985 (Fig.1); data for PV from Basso et al., 1982 (Fig.1). 
person with restricted short-term memory and normal comprehension will repeat sentences better than a person with restricted short-term memory and disturbed comprehension. Sentence span which is (partly) a consequence of sentence comprehension processes cannot be used as an explanation of disordered comprehension.

3. In comprehending the sentences from the last two parts of the Token Test, RE performed at a normal level with both auditory or visual presentation; with written sentences, though (unlike normal subjects), RE shows no effect of "articulatory suppression". Vallar and Baddeley (1987, p.437) protest that this: "suggests that her sentence comprehension processes are substantially different from those of both normal subjects and patients with defective P.S.T.S. acquired in adult age". We know of no other subject with defective S.T.M. where the effects of suppression on written sentence comprehension are investigated. Under Baddeley's (1986) working memory (W.M.) theory, suppression interferes with access to the P.S.T.S. from visual input; this predicts that there will be no effects of suppression on sentence comprehension in subjects like RE who have no P.S.T.S. Presumably Vallar and Baddeley would predict that PV, like RE, will show no effects of suppression on comprehension, because PV does not use the "articulatory loop" to recode written material into her P.S.T.S. Thus Vallar and Baddeley should be happy that RE shows no effect of suppression on Token Test performance. Their objection, presumably, is to the fact that her written sentence comprehension is normal, in the Token Test and with other kinds of material; this is because this result directly contradicts their claim that the P.S.T.S. plays a necessary role in sentence comprehension. This clearly implies that normal comprehension of long written sentences is possible without using a P.S.T.S.; normal subjects are probably affected by suppression because they persist in trying to use phonological recoding even when it is not advantageous to do so. Demonstrating that suppression has a (small but reliable) effect on sentence comprehension does not show that the P.S.T.S. is playing any essential or central role. Consider a hypothetical case: say we are interested in human locomotion. We find that subjects with their hands tied move more slowly. We find a subject with congenital lack of arms who can move at a completely normal speed. We could conclude that normal people normally walk on their hands (hence the detrimental effect of hand tying) and that the abnormal subject has learned to move in an abnormal way by walking on her feet. But these data could also be seen as showing that arms play a minimal role in locomotion (say by helping subjects to maintain their balance), and it is possible to move normally without arms by relying on legs alone.

\footnotetext{
'When tested. RE performed normally in the Wingficld and Butterworth task (B.C.H.). We argued that this was a result of her good comprehension of texi.
} 
Finally Baddeley et al. (1987, p.525) claim that the tests that we have used with RE are: "uniformly less demanding than those on which PV has been found to fail". Obviously we cannot use the identical tests since PV speaks Italian and RE English. In one case the identical test has been used in translated form; on the Token Test we used the original version, which consists of 61 commands in 5 parts (de Renzi \& Vignolo, 1964); on the final 2 parts RE scored at a level indistinguishable from controls with both auditory and visual presentation. On a shortened version with 36 items where 29 is the 5 th percentile of the normal population, PV has been tested a number of times. She scored 207 months after the onset of her illness, 23 either 14 or 23 months post onset (Vallar \& Baddeley 1984b is unclear), and 28 at the time of Vallar and Baddeley's investigations for their 1984b paper (see pp.127-129). PV's scores improve from $56 \%$ to $78 \%$ correct, and are consistently worse than $95 \%$ of the Italian population. RE only did the hardest sections of the Test and scores $95 \%$ or better, which is slightly above the average of British student controls. Thus in the only case where a direct comparison is possible between PV and RE, it is clear that PV shows a sentence comprehension problem and RE does not.

Our other tests of RE's comprehension were designed to test specific claims about the role of S.T.M. in sentence comprehension which were available at the time when we conducted our investigation; thus (Vallar \& Baddeley, 1987, p.437) we did not test "detection of anaphoric disagreement ... across sentences in passages of prose", since at that point no-one had claimed that this would be defective in a patient with defective S.T.M. However, in our sentence picture verification task we tested comprehension of sentences with embedded relative clauses (e.g. the bus that the train is preceded by is above the circle) which were multiply reversible; RE performed normally. In this test (Vallar \& Baddeley, 1984b, p.137): "preservation of the order of the words is crucial to comprehension"; in 1984 Vallar and Baddeley apparently believed that the word order information had to be held in the P.S.T.S. This test (Vallar \& Baddeley, 1987, p.432) also "demands that the subject is able to combine semantic judgement with the correct interpretation of a syntactic construction that depends crucially on the relative order of constituent words"; in Vallar and Baddeley's 1987 formulation this involves the P.S.T.S. Yet RE, who has (following Baddeley's [1986] system) no P.S.T.S., has normal comprehension of sentences of this kind.

Vallar and Baddeley $(1987, p .437)$ also claim that: "RE was not given tasks such as verification of sentences made false by a word reversal or detection of anaphoric disagreement within long and varied sentences". However the grammaticality judgements given to RE in long sentences included two types where knowledge of item order is necessary: 
1. "Functor transposed" e.g. The electricity supply failed because of two wires that have should been touching each other but weren't.

2. "Wrong voice of centre embedded relative clause" e.g. The lion who was eaten by some raw steak terrified the child.

And there are two types which involve long distance syntactic dependencies:

1. "Wrong tag" e.g. Airline pilots should never forget that the safety of their passengers is their paramount concern, ought they?

2. "Wrong reflexive" e.g. Although I never tell a lie I might in exceptional circumstances be willing to perjure ourselves.

Although these were presented in a list of long and varied sentences, RE was no worse than normal controls.

Baddeley et al.'s (1987) and Vallar and Baddeley's (1987) characterisations of our tests of RE's sentence comprehension are therefore not correct. We have, thus far, failed to find any evidence that RE has any difficulty in understanding written or spoken sentences. The Token Test results make clear that RE can understand sentences which PV cannot, despite the fact that PV's digit recall is at least as good as RE's and in some cases rather better. We cannot, of course, ever prove the null hypothesis (that RE has no sentence comprehension impairment): it is always possible that she will perform poorly on some kind of material that we have not yet tested.

Unlike PV, RE does not have an acquired cerebral lesion. Vallar and Baddeley (1987, p.436) suggest that RE may have an abnormal cognitive system: "possibly as an attempt to compensate for the deficit". PV is now ten years after onset. Vallar and Baddeley do not address the question of whether she too has acquired any compensatory strategies. Only with the Token Test do the various reports of PV provide any longitudinal data. However, her improvement from the point seven months post onset to the last test, when Vallar and Baddeley were working with her for their 1984 paper, is substantial. Some of this may be due to "spontaneous recovery", although it has generally been agreed that little or no spontaneous recovery takes place after the first six months (see, e.g. Davis, 1983, pp.213-215), but PV continued to improve long after that. It seems likely, then, that PV has acquired compensatory strategies to deal with some of her acquired deficits $^{2}$; PV's data are, from this viewpoint, neither more nor less relevant to the role of the P.S.T.S. in sentence comprehension than our data from RE.

\footnotetext{
${ }^{2}$ In no paper is there any data to show whether there has been a corresponding change in PV'S S.T.M. performance since the onset of her illness. If her S.T.M. has improved in parallel with her improved Token Test performance, the Token Test change may not only be due to the development of compensatory strategies.
} 
The data on digit recall suggest that PV has a milder short-term memory deficit than RE. The data from the Token Test show that PV has a much more severe sentence comprehension deficit than RE. There is a temptation to take this double dissociation as a demonstration that the processes involved in list recall are different from those involved in comprehension of spoken and written sentences. We think that conclusion would be too strong; however our experiments with RE have failed to find any clear evidence for a role for the P.S.T.S. in sentence comprehension. There may, however, be some kinds of sentences where the ability to hold the surface phonological form may be useful for comprehension; "garden path" sentences may be a case in point (see Butterworth et al., in press). These questions will need to be addressed on the basis of proper consideration of the available evidence.

Manuscript received 20 February 1988

\section{REFERENCES}

Baddelcy, A.D. (1986). Working memory. Oxford: Oxford University Press.

Baddclcy, A.D. \& Lewis, V.J. (1982). When does rapid presentation cnhance digit span? Bulletin of the Psychonomic Society, 22, 403-405.

Baddeley, A.D., Vallar. G. \& Wilson. B. (1987). Sentence comprchension and phonological memory: Some neuropsychological evidence. In M. Coltheart (Ed.), Altention and performance XII: The psychology of reading. London: Lawroncc Erlbaum Associates Lid.

Basso, A., Spinnler. H., Vallar. G., \& Zanobio, M.E. (1982). Left hemisphere damage and selective impairment of auditory-verbal short-term memory: A case study. Neuropsychologia, 20, 263-274.

Butterworth, B.L., Campbell, R. \& Howard, D. (1986). The uses of short-tcrm memory: A case study. Quarterly Joumal of Experimenal Psychology, 38 A, 705-737.

Butterworth. B. Shallice, T., \& Watson, F. (in press). Short-term retention of sentences without "short-term memory". In T. Shallice \& G. Vallar (Eds.). Neuropsychological impairments of short-term memory. Cambridge: Cambridge University Press.

Campbell, R. \& Butterworth. B.L. (1985). Phonological dyslexia and dysgraphia in a highly literate subject: A developmental case with associated deficits of phonemic processing and awareness. Quarterly Joumal of Experimental Psychology. 37A. 435-475.

Davis, G.A. (1983). A survey of adult aphasia. Englewood Cliffs. N.J.: Prentice-Hall.

de Renzi, E. \& Vignolo, L. A. (1964). The Token Test: A sensitive test to detect receptive disturbances in aphasia. Brain, 85, 665-678.

Pattcrson, K.E. \& Colthcart, V. (1987). Phonological processes in reading: A tutorial review. In M. Coltheart (Ed.). Attention and performance XII: The psychology of reading. London; Lawrence Erlbaum Associates Ltd.

Saffran, E.M. \& Marin. O.S.M. (1975). Immediate memory for word lists and sentences in a patient with deficient auditory short-term memory. Brain and Language. 2, 420-433.

Vallar, G. \& Baddeley. A.D. (1984a). Fractionation of working memory. Neuropsychological evidence for a phonological shortterm store. Joumal of Verbal Learning and Verbal Behaviour, 23, 151-162. 
Vallar, G. \& Baddeley, A.D. (1984b). Phonological short-term store, phonological processing and sentence comprehension: A neuropsychological case study. Cognitive Neuropsychology, $1,121-141$.

Vallar, G. \& Baddeley, A.D. (1987). Phonological short-term store and sentence processing. Cognitive Neuropsychology, 4, 417-438.

Vallar, G. \& Papagno, C. (1986). Phonological short-term store and the nature of the recency effect: Evidence from neuropsychology. Brain and Cognition, 5, 428-442.

- Wingfield, A. \& Butterworth, B.L. (1984). Running memory for sentences and parts of sentences: Syntactic parsing as a control function in working memory. In H. Bouma \& D.G. Bouwhuis (Eds.), Attention and performance X: Control of language processes. - London: Lawrence Erlbaum Associates Ltd.

\section{REFERENCE NOTES}

1. Saffran, E.M. (1985). Short-term memory impairment and language comprehension: Specifying the nature of the interaction. Paper presented at the Second Venice meeting on Cognitive Neuropsychology. 